\title{
Continuous Attachment of the Periodontal Membrane
}

\author{
ALLAN G. KRAW AND DONALD H. ENLOW \\ Department of Anatomy and The Center for Human Growth and \\ Development, The University of Michigan, Ann Arbor, Michigan
}

\begin{abstract}
A polychrome stain for differentiating precollagenous and collagenous fibers, and tetracycline for labeling bone changes were used in young rats to trace adjustments involved in movements of teeth within bone. Distinct differences were seen in fibrous attachments on depository and resorptive bone surfaces. These are associated with shifts of the periodontal membrane as the root and surrounding bone undergo drift. Some resorptive areas receive total destruction of fibrous attachment. Other areas involve a process of fibrous conversion as ordinary fibers of the bone matrix become uncovered during bone removal and function as periodontal fibers anchored into the receding bone surface. An intermediate zone of the periodontal membrane functions to progressively shorten distal ends of these same fibers and to relink them with newly formed precollagenous fibrils. These become continuous with mature fibers attached to the cementum. On depository surfaces, outer fibers of the membrane become embedded into new alveolar bone, and former precollagenous fibrils of the inward-shifting intermediate zone translocate to become the new outer zone. Continuous relinkage is simultaneously maintained between the inner and outer zones. Haversian remodeling occurs beneath resorptive surfaces and re-anchorage is thereby established in specific areas where total destruction of fibrous connection had previously occurred.
\end{abstract}

The purpose of this report is (1) to describe and interpret the response of the young, growing periodontal membrane to differential polychrome staining for collagen and precollagen; (2) to describe the nature of periodontal attachments on the various resorptive and depository surfaces of alveolar bone; and (3) to correlate the distribution of underlying Haversian remodeling, using tetracycline labeling, with periodontal anchorage on resorptive surfaces of bone.

Teeth are firmly attached to the bony alveolar wall by a dense connective tissue bridge, the periodontal membrane. Parallel bundles of collagenous fibers extend from the cementum of the tooth across the span between root and bone and are embedded in the alveolar wall. The continuous maintenance of this attachment, however, is complicated by several factors associated with growth, including differential growth rates between bone and teeth, progressive eruption, rotation and titling movements of teeth, drifting, and continuous rebuilding changes taking place in the bone of the growing maxilla and mandible. Because spatial relationships between teeth and alveolar bone constantly change during these movements, adjustment mechanisms necessarily exist that function to maintain firm and continuous attachment of the periodontal membrane to both tooth and bone. Sicher ('23, '42) reported the presence of an "intermediate plexus" in the periodontal membrane of rodents that is located in the approximate middle third of the membrane. He suggested that this plexus, containing an abundance of argyrophilic fibers, functions to splice collagenous fibers embedded in the bone on one side and the cementum on the other. The tooth can undergo eruption and at the same time maintain its attachment by a continuous resplicing of the new and old fibers as they become relocated during tooth movement. Orban ('27) later extended this explanation to human dentition. The presence of an intermediate plexus has also been reported in the guinea pig (Hunt, '59) and in the spider monkey (Goldman, '57). Several workers, however, have questioned the existence of this plexus, including Eccles ('59), Trott ('62), and Zwarych and Quigley ('65). Sicher ('65) suggests that the presence and relative prominence of an intermediate plexus is dependent upon the degree of tooth movement at the time observed. Dur- 
ing quiescent periods, this plexus presumably becomes obscured.

An important factor involved in movements of teeth within bone is the common occurrence of alveolar bone surfaces which are resorptive in character, rather than depository. This growth factor is associated with progressive movements of a tooth directly into areas previously occupied by bone. Such movements take place during both drift and eruption, and they involve the widespread resorptive removal of bone on contact surfaces between the alveolar wall and the periodontal membrane. New bone deposition occurs on the opposite (endosteal) side of the same alveolar wall. By this combined process, the entire plate of bone moves in a direction corresponding to the movement of the tooth. It is currently believed that bone resorption, in general, represents a total process of removal, including bone cells, the entire fibrous matrix, and the ground substance. It is also generally assumed that the resorption of alveolar bone, into which periodontal fibers are anchored, similarly results in a total destruction of periodontal attachment in such areas. Gottlieb ('42) has estimated that as much as 20 percent of the total alveolar bony lining can represent such a resorptive surface with corresponding fibrous detachment. Sicher and Weinmann ('44, '65) suggest that intermittent, scattered spot deposition of reparative bone on such surfaces functions to temporarily maintain partial attachment during tooth movements.

In the present report, an expansion of the concept of an intermediate plexus will be made, and additional observations and interpretations dealing with the nature of its mechanism will be presented. The critical problem of attachment and successive reattachment onto resorptive bone surfaces will be examined, and new information will be presented relative to processes involved.

\section{MATERIALS AND METHODS}

Maxillary bones from a total of $62 \mathrm{fe}$ male albino rats of the Sprague-Dawley strain were used for study. The animals ranged from 60 to 75 days in age and averaged $215 \mathrm{gm}$ in weight. Maxillary specimens were fixed in formalin aceticalcohol (10:5:85), decalcified in Decal (Winthrup Laboratories), double-embedded in celloidin, and serially sectioned at $10 \mu$. A variety of horizontal, vertical, and frontal sections were prepared. Sections were stained with a polychrome stain (Herovici, '63) to differentiate mature collagenous fibers from formative (precollagenous) fibrils. This procedure was used in order to identify and localize sites of collagen formation and to correlate their distribution with changes in the periodontal membrane and alveolar bone during drift and eruption: Using this stain, mature fibers appear red (acid fuschin), and precollagenous fibrils appear blue (methylene blue). A number of representative sections were also stained with standard Hematoxylin and eosin and with Mallory-Heidenhain for comparison with the polychrome stain.

Tetracycline hydrochloride ( $30 \mathrm{mg} / \mathrm{kg}$ body weight) was also administered to a group of 14 animals in a series of three intraperitoneal injections at 48-hour intervals. This procedure was used to identify and localize remodeling changes in the bone tissue of the alveolar wall associated with tooth movements and periodontal adjustments. Tetracycline becomes incorporated within newly formed bone, and layers or zones of such bone can be visualized by the use of ultraviolet microscopy (Behatia and Sognnaes, '63; Gregg and Avery, '64; Owen, '63; Bevelander, Nakahara, and Rolle, '60).

\section{OBSERVATIONS}

Alveolar bone surfaces and fibrous anchorage. Two general kinds of bone surfaces, depository and resorptive, line the alveolar cavity, and each is readily identifiable in routine preparations using medium power magnification. Depository surfaces are characterized by an accumulation of successive layers composed either of lamellar or non-lamellar bone tissue. As new bone is deposited, bundles of periodontal fibers become incorporated within the bone in a manner comparable with Sharpey's fibers in areas of muscle or tendon attachment (fig. 3 ). The resulting bone type is distinctive in structure and is commonly termed "bundle bone." 
Although this alveolar bone type represents the predominant bone tissue present beneath depository surfaces, some scattered areas may also be observed in which such fibrous attachment does not occur. In this instance, the only direct inter-connection between bone and the periodontal membrane is by vascular bundles and cell processes. Both types of periodontal bone, which correspond to periosteal bone tissue, are formed in areas where movements of teeth proceed in a general direction away from the bone surface. Progressive new bone deposition on the alveolar surface serves to maintain a constant boneto-tooth position as the tooth shifts in location due to drift or eruption.

Resorptive alveolar surfaces are characterized by a scalloped margin (fig. 4) and an abundant distribution of prominent osteoclasts. Such surfaces are located in areas involving progressive movement of the root in a direction toward the bone surface. The resorptive removal of bone from this outer periodontal surface, together with new bone deposition on the opposite (endosteal) side, serves to move this entire area of the alveolar wall in a direction corresponding to the movement of the advancing tooth.

In contrast with the depository surfaces just described, resorptive surfaces can be observed in which fibrous continuity with the periodontal membrane has become severed as a result of the resorptive destruction of alveolar bone tissue during tooth movements. This situation is typically seen in those areas in advance of vascular bundles as they enter the bone within formative resorption spaces (figs. $2,4)$. The resorptive surface of the bone is discontinuous with the periodontal membrane, and fibrous attachment between the two is lacking in such areas.

Another common relationship occurs between the periodontal membrane and resorptive bone surfaces, however, that has been previously unrecognized. A significant distribution of resorptive surfaces were observed in which direct fibrous continuity between the bone and the adjacent periodontal membrane was not detached or destroyed as a result of bone removal. In this situation, the movement of the alveolar wall in an endosteal direction (away from the advancing root of the tooth) leaves a trail of uninterrupted and apparently undisturbed collagenous fibers that were once a component part of the ordinary bone matrix but which had become progressively uncovered as the remainder of the bone around them was removed (fig. 4). They then become directly incorporated into the shifting and continuously reforming periodontal membrane. This observation is in contradiction with the widespread belief that bone resorption necessarily results in a total destruction of the entire bone matrix. This can be true in areas adjacent to blood vessels, as described in the previous paragraph, and in such regions bone resorption results in total fibrous detachment. In contrast, however, resorptive surfaces that are not directly associated with periodontal vascular bundles are characterized by a retention of collagenous fibers released from the bone matrix as a result of bone resorption. As they are uncovered from the receding bone surface, these bone matrix fibers become actual periodontal fibers that insert into the remaining bone and retain direct continuity with those matrix fibers still enclosed as a part of the bone tissue. Uninterrupted and continuous attachment is thereby maintained in such areas. As bone fibers are uncovered to become periodontal fibers, they subsequently undergo a process of shortening and relinkage with precollagenous fibrils within the periodontal membrane in order to accommodate membrane shifts and tooth movements. This process will be outlined in later paragraphs.

Bone matrix fibers that become exposed as a result of the removal of bone from around them are not "Sharpey's fibers" of the kind present within bundle bone beneath depository surfaces. The latter are periodontal fibers that become embedded within new bone deposits as special anchoring fibers and represent inclusions within the ordinary intercellular bone matrix. In contrast, fibers that are uncovered and preserved during resorption undergo a converse developmental sequence. They are ordinary bone fibers that subsequently become converted into periodontal fibers. Within the matrix of 
the bone, they are not arranged in coarse, parallel bundles oriented in a perpendicular plane. Rather, they enter the bone and merge directly with the general fibrous matrix (Compare fig. 3 with fig. 4 ). It is noteworthy that a comparable process of fibrous exposure, conversion, and subsequent relinkage also occurs on resorptive periosteal bone surfaces in which muscles and tendons insert (Enlow, '62b, '65b; Hoyte and Enlow, '66).

Four contrasting situations are, thus, characteristic of various depository and resorptive surfaces in contact with the periodontal membrane. These are (1) depository surfaces involving embedded Sharpey's fibers (bundle bone), (2) depository surfaces in which periodontal fibers do not become embedded during bone deposition, thereby leaving bone and periodontal membrane without local fibrous attachment, (3) resorptive surfaces involving a total destruction of fibrous connection and continuity as a consequence of bone removal, and (4) resorptive surfaces in which bone matrix fibers survive bone resorption and are retained as new periodontal fibers directly continuous with remaining alveolar bone matrix fibers. On depository surfaces, the first circumstance above has been most frequently encountered. On resorptive surfaces, both situations described are widespread and routinely seen in varying combinations. They appear approximately equal in total distribution, although quantitative determinations have not been made. As will be pointed out in later paragraphs, a number of related considerations are involved in each of the mechanisms outlined above.

Distribution of precollagen and collagen. In the descriptions that follow, the fibers of the periodontal membrane will be grouped into three component zones: the anchoring fibers of the cementum (the inner zone), the anchoring fibers of the alveolar bone (outer zone), and an intermediate zone joining the inner and outer zones figs. 2-4). The fibers of the intermediate zone will be termed linkage fibers.

The inner zone, adjacent to the root, typically contains large collagenous fibers arranged into coarse bundles which insert into the cementum. These fibers are composed largely of mature collagen and appear red with polychrome staining. The outer zone, adjacent to the alveolar wall, contains coarse fibers that pass directly into the bone. In general, the outer zone is more labile than the characteristically stable inner zone in that extensive fiber recombination is typically active in this area. Goldman ('57) has also reported the more stable nature of the inner as compared with the outer zone. In those regions of the alveolar wall involving depository surfaces and the formation of periodontal bundle bone, the fibers are colored a deep red with the differential polychrome stain. These fibers continue into the bone as Sharpey's fibers and retain their red color within the bone tissue. They represent mature collagen, and they have a large diameter and are arranged into coarse bundles.

Adjacent to resorptive surfaces, the fibers of the outer zone also stain with a red color, and they represent the mature collagenous fibers of the old bone matrix that have been uncovered during progressive resorption and which now serve as periodontal fibers. Mixed with these mature, red-stained fibers, however, are large numbers of fine, blue-stained, precollagenous fibrils that continue directly into the predominantly precollagenous intermediate zone. The fibers of the intermediate zone, thus, grade for a considerable distance into the outer zone. These overlapping precollagenous fibrils may extend close to the resorbing bone surface, and in this area of fiber convergence, direct blending and linkage of old and new fibers can be observed. On depository surfaces, an overlap between the young fibrils of the intermediate zone and the mature, coarse fibers of the outer zone is also seen, but the degree of overlap is less marked. The reason for this lies in the differing functions of the intermediate zone on resorptive as compared with depository surfaces, as described in other paragraphs. Large numbers of sizable osteoclasts are usually observed on the receding bone surface interspersed among the bundles of fibers. Using Mallory's stain, numerous, large, eosinophilic cytoplasmic granules of 
an undetermined nature are seen within these osteoclasts.

The intermediate zone spanning between the inner and outer zones ordinarily contains a few scattered, red-stained mature fibers in varying stages of recombination and shortening. The great majority of fibers observed in the intermediate zone, however, are delicate, blue-stained precollagenous fibrils. They form a relatively dense mesh and are interspersed with abundant fibroblasts and scattered vessels. The functional significance of the intermediate zone with its characteristic overlap into both the inner and outer zones will be evaluated in the later discussion.

In some scattered areas, the three-part zoning just described is not evident. Here, the majority of fibers throughout the entire thickness of the periodontal membrane are all coarse-bundled, mature collagenous fibers and respond with a typical red color to the polychrome stain. Such areas may be interpreted as stable at that particular time, and bone deposition or resorption was not then active. Since shifts of the periodontal membrane and linkage adjustments in response to bone movements were not involved, the entire fibrous component of the intermediate zone had progressed to maturity.

Remodeling changes in alveolar bone. In contrast to the unmodified and primary nature of periodontal bone beneath depository alveolar surfaces, endosteal bone tissues underlying resorptive surfaces are often characterized by marked internal remodeling changes (figs. 2, 4). Just beneath the surface, large resorption canals following the course of pre-existing primary vascular canals extend in a progressive direction deeper into the bone, thereby keeping pace with the advancing resorptive front of the entire receding bone surface. These erosion canals subsequently receive new deposits of bone within their enlarged lumina and are thereby converted into secondary osteones (Haversian systems). The fibrous matrix of these newly formed osteones is directly continuous with the fibers of the adjacent periodontal membrane. As the resorptive front (resorbing alveolar surface) continues to advance into the bone, the osteones un- dergo resorptive removal in turn, but their uncovered matrix fibers are converted into periodontal fibers retaining connection with the fibers of the remaining bone. The fibers of the Haversian system matrix, thus, are not subject to immediate destruction as the bone itself undergoes removal, and they thereby become incorporated directly as the new outer zone of the periodontal membrane as the latter follows the drift of the bone surface. The red-stained, mature fibers released from the resorbing osteones blend directly with the elongating precollagenous fibrils of the intermediate zone. This sequence is progressive, and the continued formation of resorption canals and new secondary osteones continues in advance of the resorbing surface of the alveolar wall. Such internal Haversian reconstruction deep to a resorptive surface is characteristic, in contrast to the unremodeled, primary nature of the bone beneath depository surfaces. This general process is comparable with the situation seen in long bones in which Haversian remodeling occurs within bone in relation to muscle and tendon attachment on resorptive surfaces (Enlow, '62b).

In tetracycline labeled bone specimens, the remodeling sequence outlined above is effectively demonstrated (fig. 1). Flourescence is observed (1) on the surface of the depository side of a tooth socket, and (2) within the enlarged canals and resorption spaces deep to the bone surface on the resorptive side of the socket. Using $\mathbf{H}^{3}$ Proline, Stallard ('63) also demonstrated bone deposition within the spaces of bone associated with resorptive alveolar surfaces.

Haversian reconstruction in tooth-bearing bone is most widespread beneath those resorptive surfaces in which the underlying bone is relatively thick. In some areas, the intervening wall of alveolar bone between a resorptive surface on one side and a depository surface on the other can be quite thin (fig. 4). In such locations, the remodeling sequence described above does not occur since the plate of bone is not sufficiently thick to contain large resorption canals or osteones. In these locations, the functional role of Haversian remodeling is not involved be- 
cause the relatively rapid formation and destruction of the entire thickness of the alveolar wall obviates the functional role of internal remodeling, as described below.

\section{DISCUSSION}

Movements of teeth and alveolar bone. The basic movements of teeth involve eruption and a progressive drifting in either a mesial or a distal direction depending upon species considered. The mechanism of drift functions to maintain firm contact between adjacent teeth as contact surfaces undergo wear, thereby bracing the entire dental arch. Teeth also experience tilting and rotating movements during drift and eruption. Another kind of tooth movement occurs which is not generally realized. The growth of any bone, including the mandible and maxilla, involves a process of continuous relocation of its component areas as the entire bone increases in overall size. As the various regions of a bone undergo such consecutive repositioning, the teeth located within these areas experience corresponding and simultaneous shifts in position in order to retain their relative position in the bone as a whole. This process of relocation in conjunction with bone changes due to growth contributes to the functional basis for the process of mesial drift (Enlow, '65a).

The drifting process of the periodontal membrane. The structure of the periodontal membrane provides an intrinsic mechanism by which the membrane itself can continuously move in a manner corresponding to the complex variety of tooth and bone movements outlined above. Just as both teeth and bone drift together in a horizontal plane, the intervening periodontal membrane also undergoes "drift." At the same time, it permits differential vertical movements between a tooth and the surrounding bony alveolar wall. Throughout these progressive changes, the periodontal membrane maintains firm attachment between teeth and supporting bone.

The horizontal drift of a tooth and the corresponding movements of its bony socket and periodontal membrane is schematized in figure 5. The three zones of the periodontal membrane are shown, including the inner and outer layers containing large numbers of coarse, mature fiber bundles. The intermediate zone, with its mesh of delicate precollagenous fibrils, overlaps and blends well into both the inner and outer zones. This overlap is particularly prominent between the intermediate and the outer zones. In diagrams 1 , 2 , and 3 of figure 5 , the tooth is drifting in a direction toward the right (away from the bone surface shown). Periodontal fibers that were once a component part of the outer zone have now become embedded within the periodontal bundle bone as Sharpey's fibers. As mentioned earlier, scattered areas of new bone formation can also occur in which fibers of the periodontal membrane do not become bound as inclusions within formative bone tissue, and direct fibrous connection between the alveolar wall and the membrane is lacking in such locations. The arrangement shown in the growth stages 1-3, however, is more commonly found. Progressive inclusion of periodontal fibers has occurred as new bone is laid down on the alveolar surface by the periodontal membrane. As seen in this figure, the outer zone is composed of coarse, mature collagenous fibers. This same area was formerly located as the intermediate zone of a previous growth stage. They were once precollagenous, finely-bundled fibrils formerly positioned in the intermediate zone but have become mature, coarsely-bundled fibers translocated into the new outer zone. The continuously shifting intermediate zone has moved in a direction to the right. It is composed of newly formed fibrils that represent an elongation of the fibrils of the old intermediate zone which, in turn, have become the mature, coarse fibers in the new outer zone. As the root of the tooth drifts to the right, the mature fibers of the inner zone, attached to the cementum, move to the right with the moving tooth. These fibers are stable and undergo relatively little turnover. The young fibrils of the continuously reforming intermediate zone become elongated behind the moving fibers of the inner zone, and they keep constant bond with them. The overlap between these two zones represents the constantly moving site of linkage between 
their component fibers. The entire periodontal membrane on this side of the tooth (the "tension" side) thereby keeps proportionate position between bone and cementum by its own mechanism of drifting. In this process, thus, the fibrils of the intermediate zone elongate on one side to keep pace with the fibers of the moving inner zone, and on the other side they become converted into the fibers of the outer zone which, in turn, become progressively embedded into newly forming alveolar bone.

On the opposite side of the root (the "pressure" side), an equivalent but fundamentally different process is involved. As the root drifts to the right in diagrams 4-6 of figure 5, the alveolar wall simultaneously shifts in a like direction. This is brought about by the resorptive removal of bone from this bone surface together with corresponding bone deposition on the opposite endosteal side. The proportionate thickness of the alveolar wall is thereby maintained. As previously described, inward-advancing, resorptive bone surfaces involve two general circumstances. First, some areas of surface resorption result in a total destruction of the bone, including its fibrous connection with the periodontal membrane. These areas typically occur around vascular bundles as they course into the alveolar bone from the periodontal membrane. Second, resorptive removal of surface bone in other areas leaves a distinct trail of mature collagenous fibers which were once a part of the bone matrix, prior to resorption, but which have become translocated as the fibrous outer zone in contact with the resorptive bone surface. As the root moves progressively in a direction toward the receding bone surface, a constant shortening of these same fibers occurs as they grade into the labile intermediate zone. At the same time, continued elongation of precollagenous fibrils takes place within the outer part of the intermediate zone, and these young fibrils link with the constantly shortening, mature fibers of the outer zone as the latter become successively uncovered from the bone matrix as a result of surface resorption. Whether such shortening involves an actual dissolution and subsequent reattachment of the mature fibers with precollagenous fibrils, or whether a process of progressive molecular recombination and conversion takes place is not now known. However, direct continuity can be traced between the matrix of the resorbing alveolar bone, the fibers of the outer zone, and the new fibrils of the intermediate zone. Fibrous connection, thus, is not interrupted as a consequence of surface bone removal.

The inner zone of the periodontal membrane, anchored into the cementum, is composed of mature, coarse-bundled fibers. As the root drifts, these same fibers are carried in a corresponding direction. Continuous relinkage occurs in the overlap area between the stable inner and the labile intermediate zones as the constantly reforming precollagenous fibrils of the latter layer become bonded as extensions of the inner mature fibers.

The complex sequence of adjustments involved in the drift of the membrane in a direction toward the alveolar bone surface involves, in summary, a direct conversion of former bone matrix fibers released during resorption into the new fibers of the outer periodontal zone. Since these fibers retain direct continuity with the fibrous matrix of the remaining bone deep to the resorptive surface, attachment is maintained between bone and membrane. Second, in the overlap zone between the outer and intermediate zones, a progressive shortening (or de-differentiation) of these same fibers occurs and relinkage takes place with a simultaneous elongation of the intermediate precollagenous fibrils. These newly formed young fibrils, in turn, become successively reformed and sequentially relinked with the stable fibers of the inner zone attached to the cementum of the tooth. This combined sequence of changes permits a "drift" of the entire periodontal membrane in such a manner that it maintains proportionate thickness during such movement. It provides, further, continued attachment of teeth to the alveolar wall.

The movements and changes just described represent, in part, the special mechanism adapted to the horizontal drifting of teeth within bone. Two separate and distinct processes are involved, one associated with the tension side and the other with the pressure side of a moving 
root. Differential movements between teeth and the adjacent alveolar wall, as in eruption and rotation, are also brought about by this sequence of progressive fibrous relinkage between the intermediate and the inner and outer zones of the periodontal membrane.

Another basic process contributes to the constant maintenance of tooth attachment to bone during their respective movements. This mechanism involves the continuous formation and reformation of secondary (Haversian) bone in advance of resorptive surfaces (figs. 2, 4). As a bone surface in contact with the periodontal membrane is destroyed, erosive penetration of resorption canals into the bone take place, and new bone is subsequently laid down within these enlarged spaces. The resulting secondary bone has a fibrous matrix that is directly continuous with the fibers of the outer zone of the shifting periodontal membrane and provides localized attachment between the membrane and the alveolar wall during this process of resorptive movement. As older Haversian systems are destroyed by the advancing resorptive front, fibers are uncovered from their matrix in the manner described in previous paragraphs. They become incorporated in turn into the outer periodontal zone where they undergo linkage with the fibrils of the correspondingly moving intermediate zone. As this occurs, new generations of resorption spaces develop in advance of the blood vessels entering the bone from the periodontal membrane, and new Haversian systems are then formed within these spaces. This process of Haversian remodeling functions as a reattachment mechanism in two specific instances. First, it occurs beneath those particular surface areas deep to a resorptive front in which bone removal has resulted in a total destruction of the bone matrix, including its fibers. This characteristically takes place in locations next to and on either side of periodontal blood vessels. Continuations of these vessels pass into the resorption spaces and eventually become the central blood vessels within Haversian canals following the formation of secondary osteones. The overall process provides a means by which reattachment of fibers becomes established in those local- ized areas where former attachment was destroyed. Haversian remodeling may also be involved in regions where firm fibrous anchorage was previously lacking. This situation, noted earlier, has been found in some scattered areas where formative periodontal bone does not embody periodontal fibers as it is laid down. Although not widespread in the rat, secondary bone reconstruction with Haversian system formation apparently can provide fibrous continuity between the alveolar wall and the membrane where such regions become involved in subsequent bone and tooth movements.

It is noteworthy that Haversian remodeling also occurs in long bones on those particular surfaces where muscles and tendons are attached (Enlow, '62b). Significantly, secondary Haversian systems are characteristically lacking in the bone of rats (and many other species) except in specific areas, such as the periodontal membrane, which involve resorptive surfaces in association with fibrous reattachment. In the skeleton of very young individuals, bone tissues are typically finecancellous in structure. The canals within this type of bone are large, and a process of compaction by bone deposition within these available spaces results in the formation of primary osteones (Enlow, '63). This developmental sequence functions in fibrous reattachment in a manner comparable with secondary Haversian remodeling in that both involve the deposition of new bone within large canals or spaces. In very young individuals, the distribution of primary osteones parallels that of the secondary osteones in older individuals.

\section{CONCLUSIONS}

Sections of decalcified maxillary bones from a group of young, rapidly growing white rats were stained with a differential collagenous and precollagenous polychrome stain. A second group of rats was treated with tetracycline, a vital bonemarking dye, and their maxillae were then sectioned and studied by fluorescence microscopy. The purpose of these procedures was to trace the sequence of adjustments that take place in the periodontal membrane and alveolar bone dur- 
ing facial growth and the complex movements of teeth.

The different kinds of bone surfaces lining the alveolar socket are described. Of special interest is the finding that resorptive surfaces adjacent to the root are associated with two separate circumstances. First, approximately half of the total resorptive surface area involves total fibrous destruction and loss of attachment. This occurs primarily in regions adjacent to vascular bundles within the periodontal membrane. On remaining surfaces of resorptive bone, some fibers within the original bone matrix are not destroyed but become uncovered during bone removal and are subsequently incorporated into the laterally-moving periodontal membrane. These fibers retain direct connection with the fibrous matrix of the alveolar bone and provide fibrous attachment and anchorage between the resorptive surface of the drifting bone and the contiguous periodontal membrane.

Deep to resorptive surfaces of alveolar bone, enlarged resorption canals containing blood vessels extend into the bone in advance of the resorptive front. Progressive deposition of bone within these erosion spaces takes place, and the fibrous matrix of the resulting secondary Haversian systems is in direct continuity with the fibers of the periodontal membrane. Repetition of this remodeling process occurs as resorption of the alveolar surface continues. This mechanism provides attachment and subsequent reattachment between bone and tooth in those particular areas which previously had involved total destruction of fibrous connection between the periodontal membrane and alveolar bone. As each Haversian system undergoes removal, in turn, the fibers of the Haversian matrix become released and serve as transient periodontal fibers that retain continuity with the remaining bone. Surface remnants of such secondarilyformed bone, as it undergoes progressive destruction during resorption, represent in part the localized areas previously attributed to "spot" deposition and repair during resorption.

Just as the root of a tooth and its surrounding bone undergo a process of drift, the periodontal membrane itself experi- ences corresponding movement. Drifting of the periodontal membrane involves two basic and different processes. One represents a mechanism associated with depository surfaces and the other with resorptive surfaces. In all areas, the actively moving periodontal membrane is composed of three zones. The inner zone is attached to the cementum and contains mature collagenous fibers which are relatively stable. The outer zone also contains mature, coarse-bundled fibers that are anchored in the alveolar bone as attachment fibers. The intermediate zone, comparable with the intermediate plexus of Sicher, contains an abundant distribution of young, precollagenous fibrils. Adjacent to depository surfaces of alveolar bone, these young fibrils are converted into the coarse fiber bundles of the outer zone as the entire membrane shifts and as former outer fibers become embedded into new surface bone. The young, labile precollagenous fibrils of the intermediate zone also provide continuous linkage with the mature, stable fibers of the inner zone attached to the cementum. Adjacent to resorptive bone surfaces, fibers released from the resorbing bone matrix become progressively shortened within the intermediate zone as the periodontal membrane drifts in a direction toward the receding bone surface. Continuous relinkage occurs between the newly-formed fibrils of the intermediate zone and these fibers of the resorbing bone matrix which serve as the transient outer fibrous zone of the periodontal membrane. Linkage adjustments simultaneously occur in the overlap area between the intermediate and inner zones. Actual mechanisms of linkage and subsequent re-linkage at the molecular level are not now known.

The combination of processes outlined above provides continuous attachment of the tooth to its alveolar wall during the complex variety of movements that take place as teeth undergo relocation due to maxillary and mandibular growth, eruption, drifting, rotation, and tilting.

\section{ACKNOWLEDGMENTS}

This study was supported in part by U.S.P.H.S. grant DE-01903 and by summer student fellowships awarded by the Uni- 
versity of Michigan School of Dentistry. Diagrams used in this report were prepared by Mr. William L. Brudon.

\section{LITERATURE CITED}

Behatia, H. L. and R. F. Sognnaes 1963 Tetracycline discoloration and labelling of teeth and bone. A review. J. South. California D. A., 31: 215.

Bevelander, G., H. Nakahara and G. Rolle 1960 The effect of tetracycline on the development of the skeletal systems of the chick embryo. Develop. Biol., 2; 298.

Bevelander, G., G. Rolle and S. Cohlan 1961 The effect of administration of tetracycline on the development of teeth. J. Dent. Res., 40: 1020.

Eccles, J. D. 1959 Studies on the development of the periodontal membrane: The principal fibers of the molar teeth. Dental Practit. Dent. Rec., 10: 31-35.

Enlow, D. H. 1962a A study of the post-natal growth and remodeling of bone. Am. J. Anat., 110: $79-102$.

1962b Functions of the haversian system. Am. J. Anat., 110: 269-306.

1963 Principles of Bone Remodeling. Charles C Thomas, Publisher, Springfield, Ill. 1964 A study of the postnatal growth of the human mandible. Am. J. Orthodontics, 50: $25-50$.

1965a Growth and remodeling of the human maxilla. Am. J. Orthodontics, 51: 446464.

1965b The problem of muscle tension and the stimulation of bone growth. Anat. Rec., 131: 3,451 .

Finerman, G., and R. A. Milch 1963 In vitro binding of tetracycline to calcium. Nature, 198: 486.

Goldman, H. M. 1957 Histologic structure of the attachment apparatus. Alpha Omegan, 25: 102.

Goto, S. 1952 Studien ůber die fastern des plexus intermedius der zahnwurgelhaut. Anat. Inst. Med. Fak., University of Okazama, 4: 177.

Gottlieb, B. 1942 Some histologic facts useful in orthodontic practice. Am. J. Orthodontics, 28: 167 .

Gregg, J. M., and J. K. Avery 1964 Studies of alveolar bone growth and tooth eruption using tetracycline-induced fluorescence. J. Oral. Ther. and Pharm., 1: 268-281.
Herovici, C. 1963 A polychrome stain for differentiating precollagen from collagen. Stain Tech., 38: 204-205.

Hoyte, D. A. N., and D. H. Enlow 1966 Wolff's law and the problem of muscle attachment on resorptive surfaces of bone. Am. J. Phys. Anthro., 24: 205-214.

Humason, G. L. 1962 Animal Tissue Techniques. W. H. Freeman and Co., San Francisco. 151-152.

Hunt, A. M. 1959 Description of the molar teeth and investing tissues of normal guinea pigs. J. Dent. Res, 38: 216-231.

Orban, B. 1927 Beziehungen zwischen zahn und knochen. Bewegung der zahn keime. Z. F. Anat. u. Entw., 83: 804.

Owen, L. N. 1963 The effects of administering tetracyclines to young dogs with particular reference to localization of drugs in the teeth. Arch. Oral Biol., 8-715.

Sicher, H. 1923 Bau und funktion des fixationsapparatus der meerschweinchenmolaren. $Z$. f. Stomat, 21: 580 .

1942 Tooth eruption: The axial movement of continuously growing teeth. J. Dent. Res., 21: 201-210.

Sicher, H., and J. P. Weinmann 1944 Bone growth and physiologic tooth movement. Am. J. Ortho. and Oral Surg., 30: 109.

1954 Principal fibers of the periodontal membrane. The Bur., 55: 2-4.

1965 Oral Anatomy. C. V. Mosby, St. Louis. 276-277.

Stallard, R. E. 1963 The utilization of $\mathrm{H}^{3}$-proline by the connective tissue elements of periodontium. Periodontics, 1: 185-188.

Stein, G., and J. P. Weinmann 1925 Die physiologische wanderung der Zähne. Z. f. Stomat., 23: 733 .

Tomes, C. S. 1923 A Manual of Dental Anatomy. Ed. by H. W. Tims and C. B. Henry. MacMillan Co., New York. 128-132.

Trott, J. R. 1962 The development of the periodontal attachment in the rat. Acta anat. (Basel), 51: 313-328.

Weinmann, J. P. 1954 The adaptation of the periodontal membrane to physiologic and pathologic changes. J. Oral Sur., Oral Med. and Oral Path., 8: 977.

Zwarych, P. D., and M. B. Quigley 1965 The intermediate plexus of the periodontal ligament: History and further investigations. $J$. Dent. Res., 44: 383-391. 
PLATES 
PLATE 1

EXPLANATION OF FIGURES

1 Photomicrograph of a tetracycline-injected specimen using ultraviolet light. New bone deposition (light-appearing band) occurs on the alveolar surface (A) of the tension side of the socket. In contrast, bone formation on the pressure side takes place deep to the surface within resorption spaces (B). $\times 50$.

2 The periodontal membrane is composed of an inner zone (A) adjacent to the cementum, an intermediate zone (B), and an outer zone (C) contiuous with the fibrous matrix of the alveolar wall. The bone surface seen in this section is resorptive, and resorption spaces and secondary Haversian systems (osteones) form and continuously reform (D) deep to the surface in advance of the drifting resorptive front. $\times 100$.

3 On the depository alveolar surface (tension side of socket), the inner zone (A), intermediate zone (B), and the outer zone (C) of the periodontal membrane are apparent, although the sequence of changes involved in periodontal drift differs from that on the pressure (resorptive) side. Compare with figure 5 . Note the embedded periodontal fibers within the bone. $\times 200$.

4 A thin alveolar wall separates two adjacent sockets. The bone surface on the left $(C)$ is resorptive, and that on the right (E) is depository. The inner (A), intermediate (B), and outer (C) zones of the periodontal membrane are seen. As the resorptive surface drifts toward the right, bone matrix fibers are uncovered and become the fibers of the outer zone (C). A trail of such fibers can be seen exiting from primary alveolar bone tissue as well as from secondary Haversian bone. Note that total fiber destruction and loss of attachment occurs, however, in advance of blood vessels (D). Such an area will later become partially filled with bone to become a secondary Haversian system. Note also that Haversian remodeling occurs only in the thicker areas of the alveolar wall and is absent from very thin, transient regions (compare area D with $\mathrm{E}$ ). $\times 100$. 

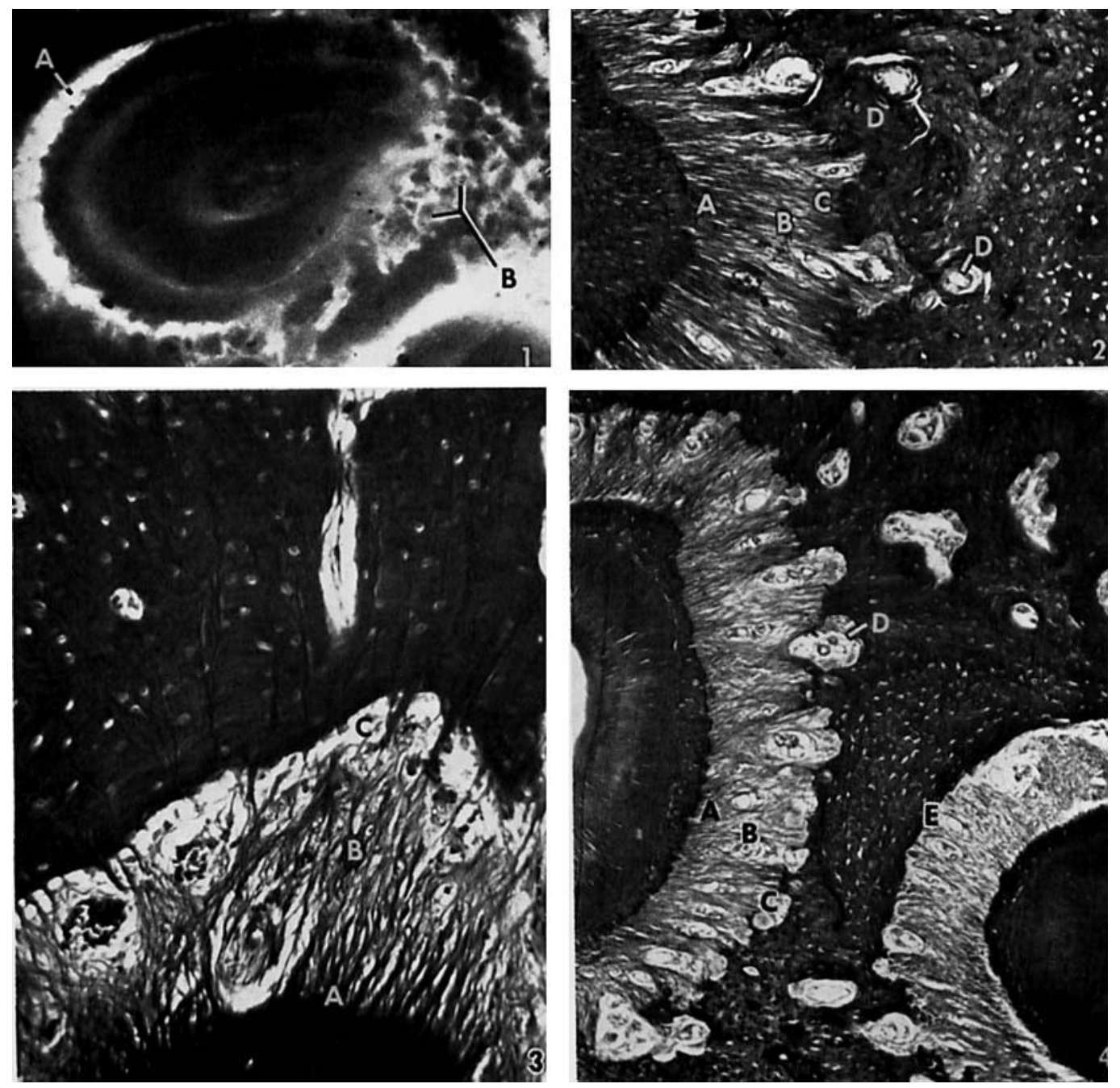
PLATE 2

\section{EXPLANATION OF FIGURE}

5 This schematic series of diagrams is an interpretation of adjustment mechanisms involved in the movements of the periodontal membrane during bone and tooth shifts. They illustrate successive and sequential changes that occur in the alveolar bone (A), the outer periodontal zone (B), intermediate periodontal zone (C), inner periodontal zone (D), and the cementum of the root (E) as both the bone and tooth drift to the right (arrows). The increment " $U$ " designates an arbitrary distance moved during each growth stage. The illustrations on the left $(1,2,3)$ represent the series of drift changes on the depository (tension) side of the alveolar socket, and the illustrations on the right $(4,5,6)$ show changes on the resorptive (pressure) side. The foints " $x$ " and " $y$ " are included as random position markers so that movements relative to a fixed point can be visualized.

On the tension side $(1,2,3)$, increments of new bundle bone are deposited on the alveolar surface, thereby progressively enclosing the fibers of the outer zone B. At the same time, the peripheral ends of the fibrils in the intermediate zone $C$ are converted into the new fibers of the outer zone as the latter shifts to the right. The stable fibers of the inner zone $D$ are pulled to the right and maintain constant linkage with the labile fibrils of the intermediate zone $C$. These latter fibrils constantly elongate in a direction toward the right as they simultaneously become converted to outer zone fibers on the left.

On the pressure side $(4,5,6)$, the stable fibers of the inner zone are moved to the right and similarly maintain constant linkage with the shortening inner ends of the fibrils in the intermediate zone. Bone-matrix fibers are constantly uncovered from the resorbing surface of $A$ to become the fibers of the outer periodontal zone $B$. The inner ends of these same fibers are then converted successively into the elongating outer ends of the linkage fibrils in the intermediate zone. Direct fibrous continuity can be traced through each zone from $A$ to $E$. Extensive bone reconstruction is seen deep to the resorptive alveolar surface, in contrast to the depository side, and resorption canals and secondary osteones are formed. This remodeling mechanism provides re-attachment in those localized areas that previously involved total resorptive destruction of fibrous anchorage. Such alternating areas are shown along the contact between $\mathrm{A}$ and $\mathrm{B}$. 
罂

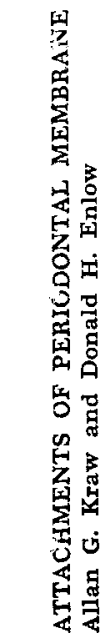
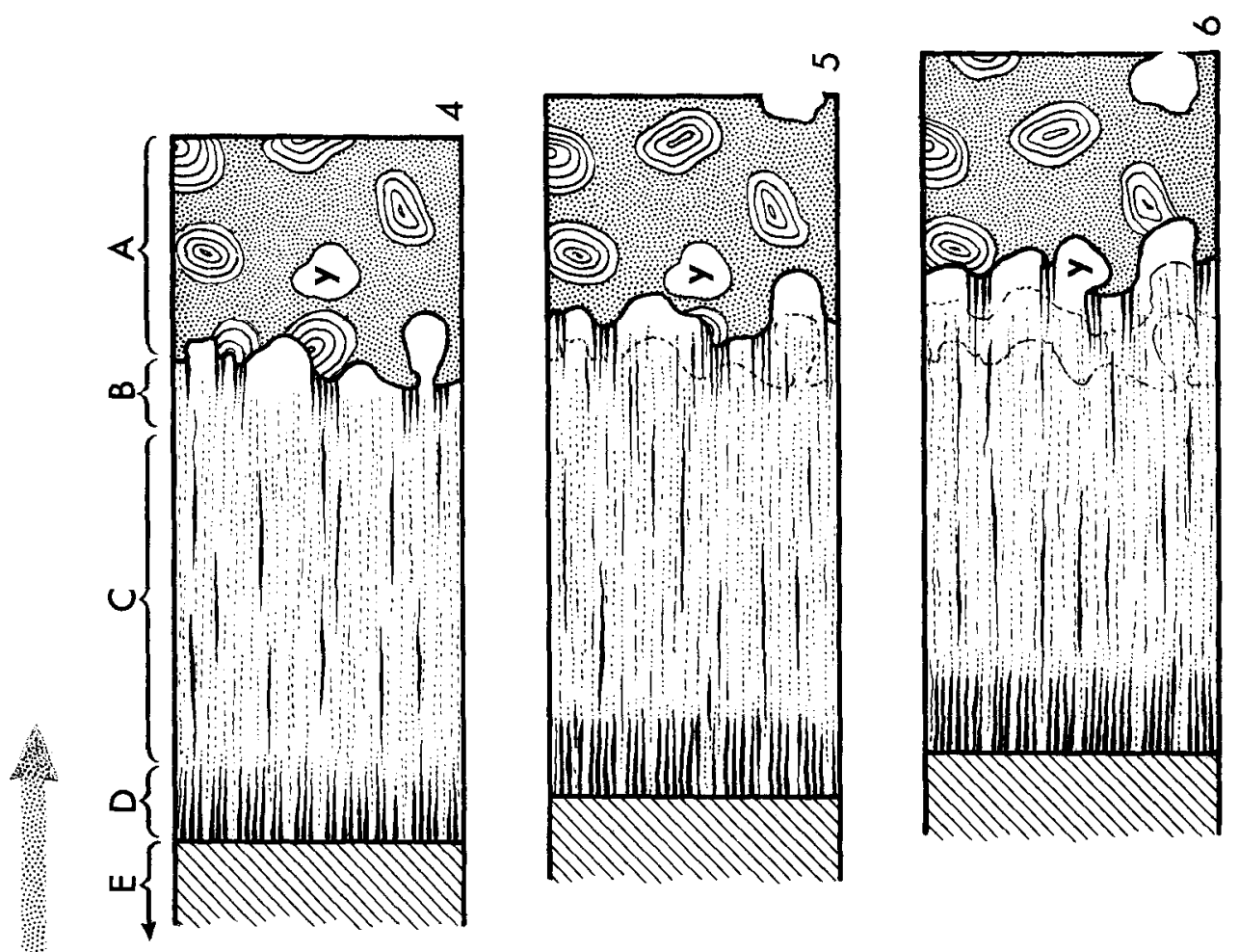

?
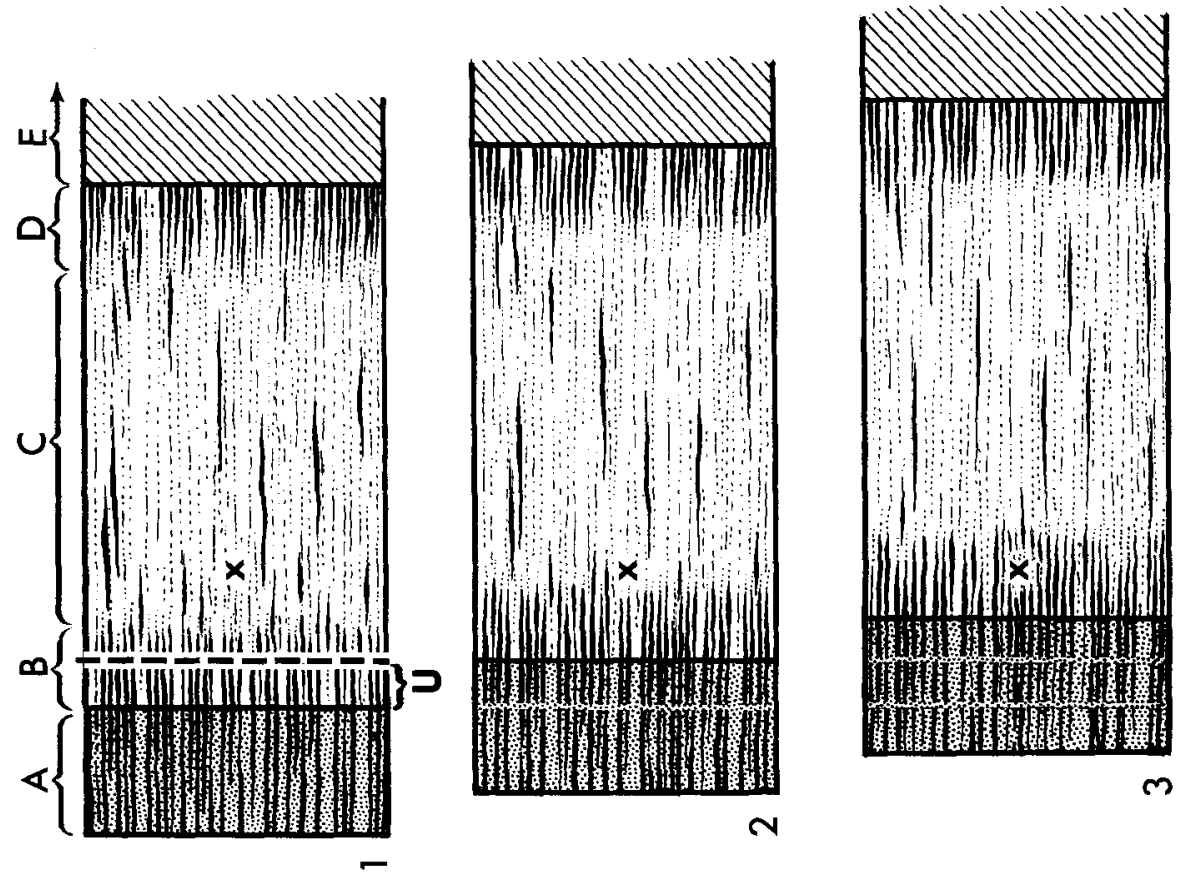